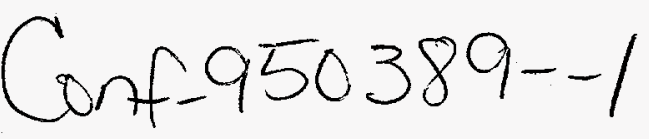

\title{
LABORATORY ROBOTICS - AN AUTOMATED TOOL FOR PREPARING ION CHROMATOGRAPHY CALIBRATION STANDARDS
}

\author{
James L. Chadwick
}

\section{DE-AC11-93PN38195}

\section{DISCLAIMER}

\begin{abstract}
This report was prepared as an account of work sponsored by an agency of the United States Government. Neither the United States Government nor any agency thereof, nor any of their employees, makes any warranty, express or implied, or assumes any legal liability or responsibility for the accuracy, completeness, or usefulness of any information, apparatus, product, or process disclosed, or represents that its use would not infringe privately owned rights. Reference herein to any specific commercial product, process, or service by trade name, trademark, manufacturer, or otherwise does not necessarily constitute or imply its endorsement, recommendation, or favoring by the United States Government or any agency thereof. The views and opinions of authors expressed herein do not necessarily state or reflect those of the United States Government or any agency thereof.
\end{abstract}

\section{NOTICE}

This report was prepared as an account of work sponsored by the United States Government. Neither the United States, nor the United States Department of Energy, nor the United States Navy, nor any of their employees, nor any of their contractors, subcontractors, or their employees, makes any warranty, express or implied, or assumes any legal liability or responsibility for the accuracy, completeness or usefulness of any information, apparatus, product or process disclosed, or represents that its use would not infringe privately owned rights. 


\section{DISCLAIMER}

Portions of this document may be illegible in electronic image products. Images are produced from the best available original document. 


\title{
Laboratory Robotics -
}

\section{An Automated Tool for Preparing Ion Chromatography Calibration Standards}

\author{
James L. Chadwick*
}

$T$ his paper describes the use of a laboratory robot as an automated tool for preparing multi-level calibration standards for On-Line Ion Chromatography (C) Systems. The robot is designed for preparation of up to six levels of standards, with each level containing up to eleven ionic species in aqueous solution. The robot is required to add the standards' constituents as both a liquid and solid additions and to keep a record of exactly what goes into making up every standard.

Utilizing a laboratory robot to prepare calibration standards provides significant benefits to the testing environment. These benefits include:

- accurate and precise calibration standards in individually capped containers with preparation traceability;

- automated and unattended multi-specie preparation for both anion and cation analytical channels;

- the ability to free up a test operator from a repetitive routine and re-apply those efforts to test operations;

The robot uses a single channel IC to analyze each prepared standard for specie content and concentration. Those results are later used as a measure of quality control.

System requirements and configuration, robotic operations, manpower requirements, analytical verification, accuracy and precision of prepared solutions, and robotic downtime are discussed in detail.

- James L. Chadwick, Sr. Scientist, Westinghouse Electric Corporation, Bettis Atomic Power Lab. P. O. Box 79, West Mifflin, Pa. 15122

\section{BACKGROUND}

Ion Chromatography (IC) was introduced in 1975 by Small, Stevens, and Baumann ${ }_{[1]}$. The technique developed in a short time, from a new way of detecting a few in organic anions to a versatile analytical technique for ionic species of all kinds. Modern IC now involves unique combinations of a number of separation systems with appropriate detectors. In 1983, Westinghouse Bettis $_{[2]}$ established a contract with Dionex Corporation to develop a twochannel on-line IC system. The base system was a $2120 i$ IC controlled with a HewlettPackard 9816 microcomputer. This automation breakthrough led to additional on-line IC systems over the next 3 years bringing the total to 49 on-line channels monitoring 37 test systems, many with multiple sample locations. A variety of chemistry matrices were being tested necessitating the need to formulate a single multi-level calibration standard containing most of the same ionic species as used in each of the test facilities. High quantities of fresh calibration standards were required to support the large number of separate on-line IC analyzers in operation. The man-power effort to support the IC calibration requirements became extensive leading to reduced test follow. To resolve this dilemma, a proposal to automate the IC 
standard preparation process by utilizing a laboratory robot was formulated. The proposal described how a robot would perform the task of preparing accurate and precise multi-level IC calibration standards, yet reflect a man power savings. In September, 1989, Zymark installed at Bettis a laboratory robot to prepare mixed anionic and cationic specie calibration standards to support continuous on-line IC operations.

\section{JUSTIFICATION AND BENEFITS OF A ROBOTICS SYSTEM}

Technical justification to procure a laboratory robotics system was very straight forward. Hazardous materials concerns or environmental effects were not an issue with this application. Listed below are the primary advantages derived as a result of installing a robotics system for standards preparation:

- The robot has the ability to operate unattended or on back shifts. In doing so a skilled technician has been relieved of a boring repetitive job to make more efficient use of his time for test preparation and test follow.

- The problem of technicians' busy schedules interfering with preparation of standard solutions has been eliminated. QAVQC requirements were jeopardized because calibration of equipment could not be maintained without fresh calibration solutions.

- The quality of calibration standard has improved by eliminating gross error, occasionally encountered in human prepared standards (i.e.. missing ionic species, several fold error in concentration).

- With each standard solution prepared a record of its contents is printed for QA/QC traceability. The robot also maintains record of analytical verification for each standard.

\section{DEFINING THE ROBOT OPERATING PRO- CEDURE}

Once justification for need was established the question of robot capability required investigation. The system would be required to prepare standard solutions with precision and accuracy equivalent to that of a technician or scientist.

The ideal role of the robot would be to prepare multi-level mixed anion/cation calibration standards in volumes that are easy to handle by man and robot. The system must be capable of maintaining records of the contents, final dilution, and analytical results of each prepared standard. In addition, the system must be capable of either flagging the operator of an improperly prepared solution, or, discard or isolate it for proper disposal.

To accomplish this, a step by step list of actions or LUO's, Laboratory Unit Operations $s_{\{3\}}$, had to be created to determine exactly what the robot could or could not do based on the ideal role described above. At this time operational checks and calculations were not considered because they would be addressed during debugging. 
To determine a list of LUOs required for this operation, a flowchart was prepared to show exactly how a standard solution would be prepared as if it were being prepared by man. This also helped in determining if precision and accuracy requirements could be met. The laboratory procedure of preparing IC calibration standards would then be systematically translated ${ }_{[4]}$ into terms of:

- The required LUO

- Their sequence in the procedure

- What happens in each LUO

Listed below are the LUOs based on manmade standard preparation.

- Manipulation (fleakers)

- Liquid Handling (add water and preservative to fleaker)

- $\quad$ Conditioning (stirring)

- Manipulation (weigh dish on balance pan)

- Weighing (weigh powder)

- Manipulation (powder dumped into fleaker)

- $\quad$ Conditioning (stir fleaker to mix)

- Liquid Handling (add reagents and final dilution)

- $\quad$ Conditioning (stir to mix)

- Manipulation (move fleaker to ion chromatograph sampler)

- Measurement (ion chromatograph analysis )

- Manipulation (return fleaker to storage)

- Documentation (standard identification and analysis results stored)

This LUO list along with detailed documentation on additional required robotics operations was sent to Zymark Corporation for logic review and parts availability. Zymark replied favorably with respect to off the shelf hardware, pysections, and equipment. A pysection is robotic hardware integrally designed to perform some LUO and is attached to key reference points on a circular mounting plate. All standard operating commands for each pysection are preprogrammed on an accompanying disc using Zymark's EASYLAB software.

There were two sections that required custom manufacture and fit. This meant the final system would have to be set up and tested at the vendor's assembly facility prior to shipment. Due to Westinghouse Bettis policies only the custom pysections could be tested and debugged at Zymark. The remaining setup and testing was completed on site by Zymark without significant problem.

\section{SPECIAL DESIGNED PYSECTIONS}

Two robot pysections were designed, assembled, and top level programmed in $E A S Y L A B$ pytechnology control language specifically for this robotic application. First, a barcode reading station was designed to read a barcode label placed on the side of a fleaker. The barcode label is a single 6 digit number placed on the side of a fleaker that the scanner and robot controller interprets as: 1) a standard identification used for traceability, and 2) an identifier that directs the robot to what calibration level to prepare. The numbers range between 100000 to 699999 incremented singly. Each incremental range of 100000 relates to a calibration level (i.e. 100000 thru 199999 for level one, continuing respectively up to 699999). The section was designed to contain a rotating platform and a $1.0 \mathrm{mw}$ Class IV laser scanner. The platform rotates a fleaker (placed there by the robot) enabling 
the barcode label to be scanned by the laser reader. Once the label is read and information is stored in the controller, the fleaker is removed from the platform. The second, is a variable speed magnetic stirring section. This section was designed to mix and dissolve all additions to the 300 $\mathrm{mL}$ fleaker. Both pysections would be controlled through relays and power switches from a Power and Event Controller (PEC).

\section{ORIGINAL ROBOTICS SYSTEM}

The original robotics system required several pieces of additional equipment not available from Zymark. Below is a list of that additional equipment:

Balances - Mettler AE200 with communications interface

Mettler PM400 with communications interface

Barcode Scanner/Reader - Symbols Technologies

Fleakers \& Caps - Caps from Zymark Test Tubes - 30mm X $150 \mathrm{~mm}$ Vials with Caps - $30 \mathrm{~mm}$ X $150 \mathrm{~mm}$ Reservoirs - $1 \& 2$ liter plastic

The pysections originally procured have remained unchanged. Upgrades to the robot arm, controller, and computer system have been installed several times since 1989 . Listed below is a list of pysections from the original system that are still in use today:

Fleaker Rack(2) - 300 ml. size

Barcode Reader

Balance Section (PM400)*

Magnetic Stirrer
Test tube Rack ( $50 \mathrm{~mm} \times 150 \mathrm{~mm}$ size tubes)

Cap/Uncap Station

Balance Section (AE200)

Waste Chute

Hand $\mathrm{C}$

Hand $G$

Hand $\mathrm{E}$

Master Lab Station (2)

Power and Event Controller (2)

Zymate II Robot Arm

Controller, Disc Drive, and Monitor

* One balance section was modified to mount an air operated arm holding six liquid delivery lines that would swing over the fleaker (while on the balance) and deliver pre-determined volumes of liquid. It also contains a small funnel attached to a drain that allows flushing of the lines while the arm is in the rest or retracted position.

This system operated until the System V controller and PC computer interface was introduced about 2 years later.

\section{SYSTEM UPGRADES TO DATE}

The program and dictionaries to operate this system required over half the available memory of the Zymate II controller. The first major upgrade after about 2 years of operation was to the System V controller and $\mathrm{PC}$ interface. This improved process speed and made available additional memory.

The second major upgrade in December, 1993, was the addition of a new PC interface system and high speed XP Arm. This made a difference of $30 \%$ less in preparation time of a single solution. Arm position calibrations are maintained longer 
controller, IC computer, and Dionex IC

2) Robotic Operations -- commencement of operations was designed to use a single command. By typing 'ROBERTA' and answering all remaining questions, using the computer keyboard, the robot would begin preparing calibration solutions. The following is the current step by step robot procedure to prepare a single IC calibration standard:

- uncap the first fleaker in line and store the cap in the holder - remove the fleaker from the rack and place it on the turntable for the barcode reader

- the fleaker is rotated several times, the laser scans the barcode and reads the number

- the fleaker is transferred to the PM400 balance for the addition of 150 grams of DI water. During the addition of water, the robot's hands are switched and a $0.2 \mathrm{~mL}$. aliquot of $4 \%$ formaldehyde is transferred to the fleaker. Hands are changed back and the robot arm waits until the fleaker has received the 150 grams of water before continuing - the fleaker is transferred to the magnetic stirring bar section to start mixing -the robot changes hands to pick up an uncapped glass vial from $R A C K .3$ and places it in the AE200 balance - the robot then picks up the capped vial containing the dry chemical specie from the same rack - the vial is uncapped and the cap stored on one of the storage pedestals on the pysection - the robot adjusts the tube from a picking up grip to a pouring grip and moves over top of the test tube in the
AE200 balance

- based on the barcode numbering sequence the controller has stored the target value of powder to add to the tube. The robot now pours from the tube using the vibration option in the hand to slowly pour powder into the test tube. The balance continually takes weight readings until the actual weight is within $+/-11 \%$ of the target weight

- once the target weight has been satisfied the powder is transferred into the stirring fleaker - the robot changes hands and transfers the fleaker to the PM400 or addition of remaining ionic species and final dilution water - the ionic species are added by a Master Lab Station using 5 and 10 $\mathrm{ml}$. syringes. (NOTE: The volumes added are dependant upon the $+/-$ difference of the actual powder weight in relation to the targeted weight. The difference is automatically calculated and adjustments are made in all remaining additions so that all standards of the same level are prepared to the same oncentration.) Frequently, final volumes differ as much as $75 \mathrm{~mL}$. - the fleaker is transferred back to the stirrer for final mix - the fleaker is transferred to the IC sipping station where a sample is drawn into the IC via a master lab syringe. The IC then analyzes the prepared solution.

- the fleaker is returned to it's original position along with its cap - the robot then moves onto the next fleaker, provided one exists. If not the arm parks the hand and the system rests in an idle position. 
and frequency of routine adjustments have been reduced from monthly to quarterly. This upgrade also resulted in a reduction of preparation errors by at lest $50 \%$.

The last upgrade included the addition of Concurrent programming capabilities. This enabled non-robotic operations (i.e. liquid dispensing, weighing, or any operation not using the robot arm) to be performed simultaneously with robotic functions. A Dionex DX-100 single channel (anionic) ion chromatograph system was added to support the actual analysis of every standard solution prepared by the robot. It should be noted that reagents were chosen to utilize both the anion and cation in the standard matrix. For this reason, it was determined that only anion checks of the prepared standards were needed. A Zymark $L C / I C$ Sipping Station was also added to support the IC system.

This final and current configuration of the robot system is shown below in the overhead . outline layout.

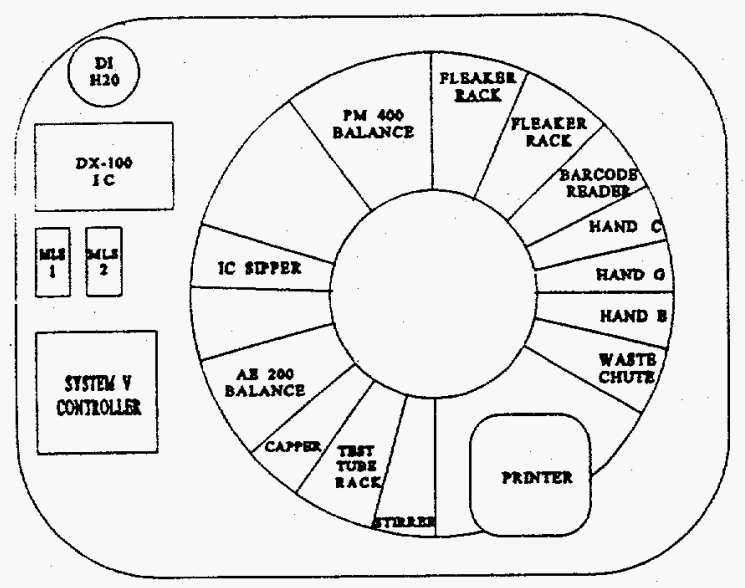

The System V Controller, PC system, and printer are located on an adjacent bench top.

\section{DETAILED SYSTEM OPERATION}

After determining the proper Robotic LUOs' the system programming was assembled in modules. Most pysection command modules, (i.e. Get From Rack.5) required some modification for smooth and efficient operation. The operating procedure to prepare single or multiple IC standard solutions was broken into two steps: Manual Operations and Robotic Operations.

1) Manual Operation -- the following must be performed prior to each robot operating session. Corrections to each operation are to be completed only if necessary:

- the level of solution containers must be $>1 / 3$ full to insure adequate supply for the operating session - the level of dry chemical in each vial must be $1 / 4$ to $1 / 2$ inch deep to allow for disbursement from the test tube - all fleakers have been barcode labeled are capped

- check to ensure a magnetic stirrer is in each fleaker so that mixing of all reagents is complete - check for at least $75 \mathrm{~mL}$ of $<14$ day old $4 \%$ formaldehyde solution is available so that the correct amount of fresh preservative is added - a full rack of 10 clean empty test tubes

- sufficient quantity of pipette tips for at least twice as many standards being prepared

- the printer is on-line and gas pressure at 25-30 psi. to prevent malfunction of air-operated components

- power on the robot computer 


\section{PRECISION OF}

\section{PREPARED}

\section{CALIBRATION SOLUTIONS}

Shown below are graphs reflecting the results of a group of standards analyzed by the DX-100 IC. Each graph reflects the precision of a specie from one run to another. It is important to note that the precision of the IC analysis has not been factored out of these graphs. The results shown give a more realistic number to the precision of the overall analytical results of actual test samples. Calibration of the DX100 IC was performed each time the robot ran using diluted NIST Anion Standards.

Spacis "A" Concentsatton

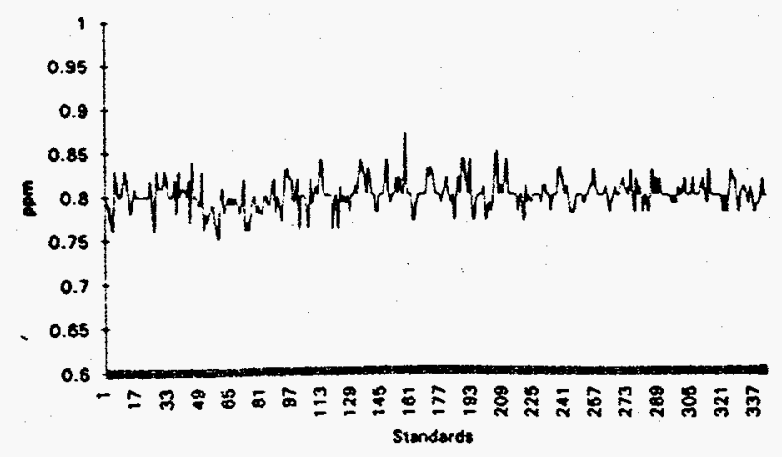

Specie "8* Concentration

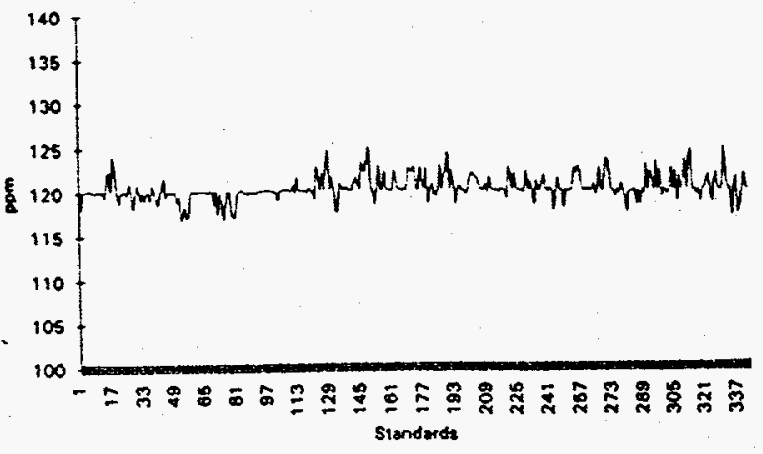

$\begin{array}{ll}\text { Theoretical Standard Value } & 120.00 \mathrm{ppm} \\ \text { Average Standard Concentration } & 120.30 \mathrm{ppm} \\ \text { Standard Deviation } & 1.35 \mathrm{ppm} \\ \% \text { RSD } & 1.12\end{array}$

Specte " $\mathrm{C}$ " Concentration

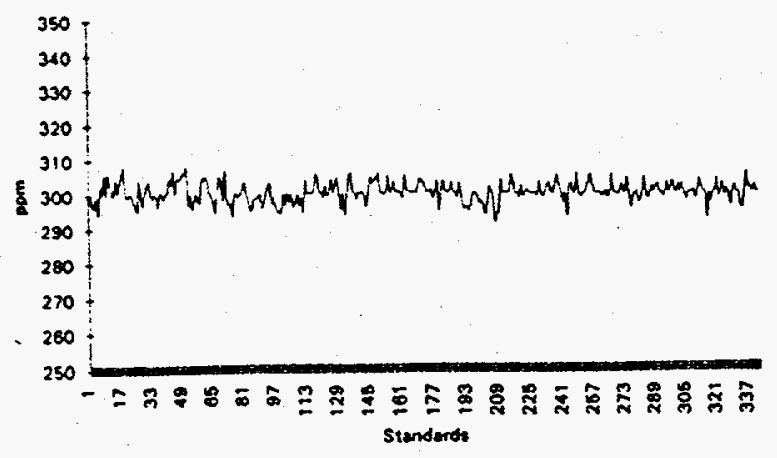

$\begin{array}{ll}\text { Theoretical Standard Value } & 0.80 \mathrm{ppm} \\ \text { Aterage Standard Concentration } & 0.80 \mathrm{ppm} \\ \text { Staodard Deviation } & 0.02 \mathrm{ppm} \\ \% \text { RSD } & 2.17\end{array}$ $\begin{array}{ll}\text { Theoretical Standard Value } & 300.00 \mathrm{ppm} \\ \text { Average Standard Concentration } & 300.13 \mathrm{ppm} \\ \text { Standard Deviation } & 2.86 \mathrm{ppm} \\ \% \text { RSD } & 0.95\end{array}$ 


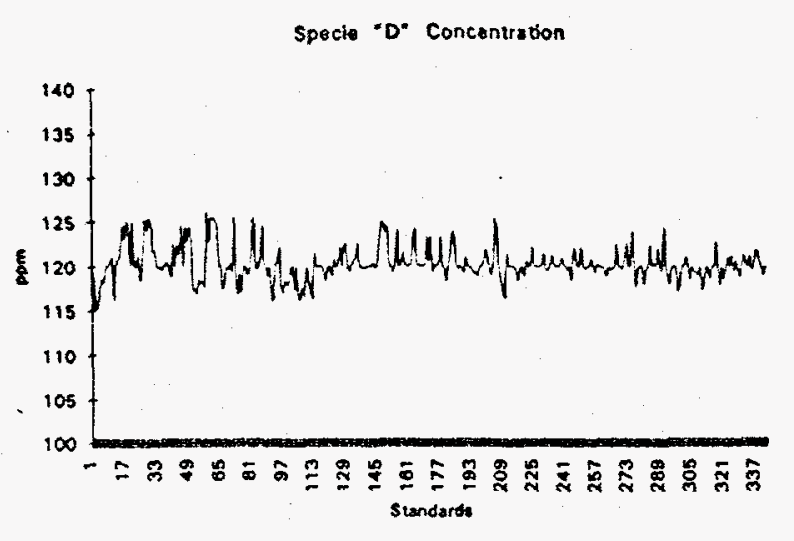

$\begin{array}{ll}\text { Theoretical Standard Value } & 120.00 \mathrm{ppm} \\ \text { Average Standard Concentration } & 120.29 \mathrm{ppm} \\ \text { Standard Deviation } & 1.96 \mathrm{ppm} \\ \text { \% RSD } & 1.63\end{array}$

Ory Specie Concentration

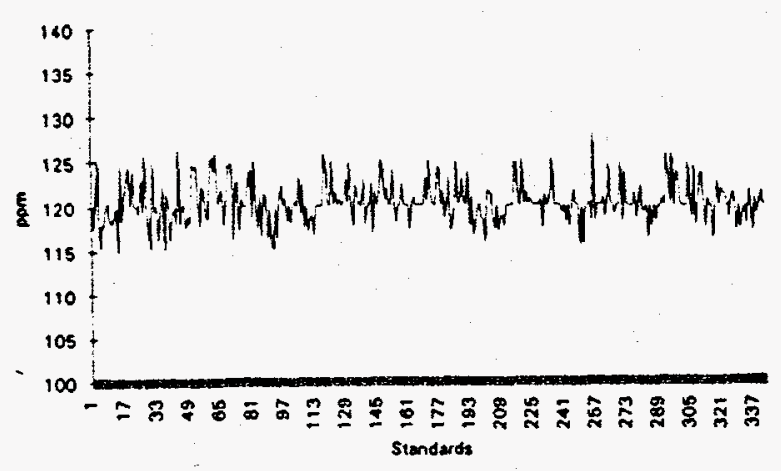

Theoretical Standard Value Average Standard Concentration

Standard Deviation $\%$ RSD

$120.00 \mathrm{ppm}$ $120.36 \mathrm{ppm}$ $2.36 \mathrm{ppm}$ 1.96

\section{BUILT IN \\ OPERATIONAL CHECKS AND CALCULATIONS}

During the procedure, checks to insure proper systematic operation and to prevent inadvertent malfunctions had to programmed into its operation. Any error in the preparation of a standard will jeopardize the validity of the robot operation along with the IC analytical method.

Barcode Reader Check - the barcode reader makes three attempts at reading the applied barcode before ending the operation with an error message and exiting from the program. Clear and legible barcodes are required to prevent improper identification of a standard level.

Water addition timer - Due to an unidentifiable communications error between the PM400 balance and the System V controller, water over additions to the extent of flooding the balance have occurred, leaving the balance inoperable. A programming timer was built into the main program to halt dispensing of water after a pre-determined length of time. This enables the robot to continue preparing the standard. The robot automatically takes into account the water over addition and adjusts all further additions accordingly. This also eliminates possible damage to equipment due to flooding.

Powder Weighing Checks - The powder is weighed into test tubes to a target value based on the calibration level (barcode number). If the powder weight is $>11 \%$ of the target value, the total adjusted volume in 
volume of the fleaker as well as the range of the PM400 balance. In such a case the robot will discard the test tube and its contents to waste. This check is in the event of over addition of powder to the test tubes during the weighing process.

A second weight check is made when the powder is actually poured into the fleaker. A weight reading is taken after the powder is transferred to the test tube and is recorded as a tare weight. A second reading is taken on the test tube after the powder has been transferred to the fleaker. The difference from the second to first is the actual weight of powder added. It is this actual weight that is used as a basis in determining volumes of other species added and the final dilution volume of DI water. If this weight exceeds the target range by $>11 \%$, the fleaker is returned to the storage rack and no other ionic species are added.

Standard IC Analysis Check - A Dionex DX-100 single channel Ion Chromatograph system checks each standard prepared for anionic contents. The results of these analyses are stored in the Dionex AI-450 software for future reference, in addition to a . hard copy chromatogram that can be used to visually inspect the chromatography of each prepared standard. The robot prints out a summary report showing the exact volumes of stock solutions added to each fleaker. By using both IC results and robot summary report, verification of the prepared standards can be easily made. These results are stored permanently per laboratory QA/QC requirements.

\section{STANDARD TRACKING AND RECORD KEEPING}

\begin{abstract}
All prepared calibration standards are controlled by barcode number and preparation date. Two separate reports are generated during each run of standards. One report lists the mass contents of each standard along with operator's name, and date of preparation. The second report is the ion chromatography results of each prepared standard solution. The two reports are coordinated by pre-written IC schedule files that match the order in which the numbered fleakers are placed in the storage rack. The robot report displays the volume of each of 4 liquid ionic additions, weight of powder added, and total volume of water added during preparation for each solution. This information combined with the preparation date, operator's name, and identification gives the operator all the information needed to correctly evaluate the standard preparation. The IC report is a verification that the solutions were prepared properly and a means to monitor deviations that occur during solution preparation.
\end{abstract}

\section{ROBOTIC, DOWNTIME, SERVICE, AND MAINTENANCE}

The robot is covered by a service contract with Zymark Corporation for quarterly preventative maintenance( $\mathrm{PM}$ ) visits and repair or full replacement of all malfunctioning components. Unexpected failures requiring a service call have occurred on the average of twice per year. Minor 
repairs not affecting quality of calibration solution preparation are delayed until a PM visit. The frequency of PM visits has ensured a very dependable system supporting continuous on-line IC operations.

The DX-100 IC system is serviced separately by Dionex Corporation with a PM schedule of 5 visits per year.

Balances are cleaned and calibrated quarterly by a qualified vendor.

Overall daily manpower support is very minimal. The primary operator effort required to prepare the robot for operation is mostly used for cleaning fleakers and preparing them for use. This is a daily requirement and usually is completed in about 15 minutes for a run of 12-14 standards. Every other month the dry powder is replaced with fresh reagent grade material and the solution reservoirs are refilled. The pipet tip rack is refilled monthly.

\section{SUMMARY}

The robot has proven to be a vital asset to the test laboratory. Its reliability of operation has never been a problem. The quality of standards prepared is reflected in the high quality of On-Line Ion Chromatography data produced daily. It has enabled an increase in test productivity over the 5 years of operation by saving and redirecting a total of about 1.2 man years. The robot was relocated in May, 1994 to a permanent private room so its environment can be better controlled and monitored. The future of this system is promising. Plans are to keep it operational daily, well into the 21 st. century.

\section{ACKNOWLEDGEMENTS}

A special thanks and appreciation are extended to:

Jack Carr -- Westinghouse Bettis

Ron Briggs -- Zymark Corporation

Dale McBride -- Dionex Corporation

\section{REFERENCES}

[1] Joachim Weiss, Germany: "Handbook of Ion Chromatograghy", Dionex Corporation, Sunnyvale, California

[2] Gary Lynch, "Practical Experience With OnLine Chromatography", Process Control and Quality, 1(1991) 249-263, Elsevier Science Publishers B.V., Amsterdam

[3] Zymark Corporation, "LABORATORY ROBOTICS HANDBOOK", (1988)pgs 18,20, Zymark Corporation, Zymark Center, Hopkinton, Mass., 01748

[4] Zymark Corporation, "LABORATORY ROBOTICS HANDBOOK", (1988)pgs 20, Zymark Corporation, Zymark Center, Hopkinton, Mass.,01748 